\title{
The human intestinal microbiota and its relationship to energy balance
}

\author{
Fredrik Bäckhed*, Ruth E. Ley, Justin L. Sonnenburg and Jeffrey I. \\ Gordon
}

Center for Genome Sciences, School of Medicine, Washington University, St Louis, Missouri, USA

\begin{abstract}
The human gut microbiota can be pictured as a microbial organ placed within a host organ: it is composed of different cell lineages that have the capacity to communicate with one another and with the host. One major function of the microbiota is to degrade complex and otherwise indigestible components of the diet, such as polysaccharides. This process results in production of short-chain fatty acids that are readily absorbed and used as an energy source by the host. Studies in gnotobiotic mouse models are providing new details about how the gut microbiota can affect how calories from the diet are harvested and stored.
\end{abstract}

Abbreviations: Angpt14: angiopoietin-like protein 4; anti- $\sigma$ : anti-sigma; ECF- $\sigma$ : extracytoplasmic function- $\sigma$; Fiaf: fasting-induced adipocyte factor; GF: germ free; Lpl: lipoprotein lipase

\section{Introduction}

$\mathrm{F}$ rom the time of birth to the moment of death, the human body provides a home for microorganisms. As adults, the number of microbes associated with body surfaces is estimated to exceed the number of human cells by $\sim 10$-fold $(1-3)$. Thus, a broad and encompassing view of humans as a lifeform requires an understanding of the contributions of these indigenous microbial communities to human biology.

The intestine contains the largest collection of microorganisms: some 500-1000 different species of Bacteria and Archaea (3-5), although marked differences in species composition exist between individual people (4). Regardless of interpersonal differences, however, the vast majority of bacteria ( $>90 \%$ ) detected in humans belong to two phylogenetic divisions of Bacteria: the Bacteroidetes and the Firmicutes. Aside from these predominant divisions, rarer members belong to eight other divisions (out of 70 described: Actinobacteria, Proteobacteria, Verrucomicrobia, VadinBE97, Spirochaeates, Synergistes, Cyanobacteria and Fusobacteria) $(3,4)$. The Archaea native to the human gut are less abundant and less diverse than the Bacteria, and are typically represented by one

\footnotetext{
* Present address: Wallenberg Laboratory, Sahlgrenska University
} Hospital, Göteborg University, Göteborg, Sweden. dominant kind, the methane-producing Methanobrevibacter smithii (4).

The collective genomes of these gut microbial species (the microbiome) are thought to possess around 100-fold more genes than the human genome (6), and endow humans with metabolic capabilities that they have not had to develop on their own, including the ability to harvest nutrients from varied diets that would be otherwise indigestible, e.g. plant polysaccharides (3).

To understand better the foundations of humanbacterial symbioses in the intestine, (i) the genomes of prominent members of the human gut microbiota were sequenced, (ii) germ-free (GF) normal or genetically engineered mice were colonized with single or defined collections of (sequenced) microbial species, and (iii) the effects of colonization on host and microbial biology were observed using functional genomics, mass-spectrometry-based metabolomics and other physiological profiling methods (e.g. 7-12).

\section{A prominent member in the human gut microbiota that provides nutrient-processing capabilities that humans have not evolved on their own}

Bacteroides thetaiotaomicron harbors a very large arsenal of genes involved in sensing, acquiring and metabolizing nutrients. Its environmental sensing 
apparatus includes 50 extracytoplasmic function- $\sigma$ $(\mathrm{ECF}-\sigma)$ factors and 26 anti-sigma (anti- $\sigma$ ) factors, 79 members of classic two-component systems, and 32 novel hybrid two-component systems that incorporate all of the domains encountered in a twocomponent system into a single polypeptide. Nutrient acquisition appears to be mediated, at least in part by 209 paralogs of two cell-surface proteins that bind starch (107 paralogs of SusC; 102 paralogs of SusD). Its glycobiome also contains 226 predicted glycoside hydrolases and 15 polysaccharide lyases (11). In contrast, the Homo sapiens genome, which has a coding potential approximately five times that of B. thetaiotaomicron, specifies fewer than 100 of these glycan-degrading enzymes. Identification of the more than 60 enzymes in the $B$. thetaiotaomicron genome that can be definitively assigned to degradation of plant/dietary polysaccharides (dietary fibers; e.g. xylans, arabinogalactans, pectin) underscores the complementary metabolic capabilities that this symbiont confers upon its human hosts, who encode only one putative enzyme in this class (http://afmb.cnrs-mrs.fr/CAZY) (12).

The regulation of the transcriptome of $B$. thetaiotaomicron was studied in vivo by analyzing its global gene expression profile in the ceca of NMRI mice that were colonized for 10 days and fed a polysaccharide-rich diet or a simple-sugar diet (12). These studies revealed that $B$. thetaiotaomicron monitors glycan availability in the gut and prioritizes its nutrient harvest, focusing primarily on dietary plant polysaccharides. When plant polysaccharides are removed from the host diet, $B$. thetaiotaomicron has the capacity to subsist on glycans derived from the host, e.g. mucus (12). The adaptive foraging behavior exhibited by $B$. thetaiotaomicron in vivo is facilitated by its expanded families of genes encoding glycan importing and degrading machinery: these genes are physically linked to genes encoding nutrient sensors in polysaccharide utilization clusters. This arrangement provides insights into the strategies evolved by a successful human gut symbiont so that it can persevere in the highly competitive and dynamic distal gut environment.

\section{The gut microbiota regulates adiposity, in part through regulation of expression of a gut epithelial protein that is a circulating inhibitor of lipoprotein lipase}

By comparing adult GF C57B1/6J mice with (i) conventionally raised mice (i.e. animals that acquired a microbial community beginning at the time of birth) and (ii) mice that were first raised to adulthood in a germ-free state and then colonized with a complete cecal microbiota harvested conventionally raised donors (a process termed conventionalization), it was found that conventionalization produces a $60 \%$ increase in body fat content, and relative insulin resistance within 14 days, despite reduced food intake and energy expenditure (10). The increase in total body fat content after a 14 day conventionalization was equivalent to the difference in fat content between adult GF and conventionally raised mice. The fat storage phenotype was not unique to the C57B1/6 $\mathrm{J}$ inbred strain: it was also observed 14 days after conventionalization of 8-week-old NMRI mice (10). More prolonged colonization did not produce a further increase in the amount of deposited fat conventionalized animals (10). Microbial fermentation of dietary polysaccharides to short-chain fatty acids in the distal gut and their subsequent absorption stimulate de novo synthesis of triglycerides in the liver through effects on two basic helix-loophelix/leucine zipper transcription factors: Chrebp and Srebp-1c (10). The augmented epididymal fat pad weight in conventionalized mice is due to hypertrophy rather than to hyperplasia, and is accompanied by increased lipoprotein lipase $(\mathrm{Lpl})$ activity (10). Fasting-induced adipocyte factor (Fiaf; also known as angiopoietin-like protein 4, Angpt14) is a recently described secreted Lpl inhibitor (13). Colonization of the gut is associated with a diminution of small intestinal Fiaf expression, without changes in its expression in liver or white adipose tissue. Moreover, studies of GF Fiafdeficient knockout $\left(\right.$ Fiaf $^{-1-}$ ) mice revealed that they have $122 \%$ higher white adipose tissue Lpl activity, and the same amount of total body fat as their age- and gender-matched conventionalized (Fiaf-suppressed) wild-type littermates (10). In addition, a 14 day conventionalization of GF Fiafdeficient knockout mice produced no substantial further increase in their total body fat stores $(\leq 10 \%$ compared with the $55 \%$ increase in their Fiaf $^{+1+}$ littermates) (10).

\section{Obesity alters gut microbial ecology}

16S rRNA gene sequencing was used to enumerate the distal intestinal microbiotas of genetically obese $L e p^{o b} / L e p^{o b}$ mice, and their lean $L e p^{o b /+}$ and wildtype siblings, plus their $L e p^{o b /+}$ mothers. All mice 
were fed the same diet; littermates were separated at weaning and maintained for 5 weeks in separate cages. The results revealed that: (i) animals inherit their microbiota from their mothers; (ii) community membership is remarkably stable over time; and (iii) obesity alters microbial community structure (the relative abundance of members): compared with lean mice and regardless of kinship, obese animals have a $50 \%$ reduction in the abundance of Bacteroidetes and a proportional increase in Firmicutes (5). These changes are division-wide. The underlying mechanism is not known and is not attributable to differences in the amount of food consumed by obese versus lean animals (5).

Ongoing metagenomic studies are characterizing the microbiomes of lean and obese mice. The results should provide information about whether these observed changes in microbial ecology are a factor contributing to obesity, or an adaptive mechanism designed to diminish the capacity to harvest energy from the diet of a host that is obese.

The results may provide new microbiota/microbiome-targeted therapeutic approaches for insuring proper energy balance.

\section{Acknowledgements}

We thank Peter Crawford and Clay Semenkovich for helpful discussions. This work was supported in part by NIH grant DK70977. FB is the recipient of a postdoctoral fellowship from the Wenner-Gren Foundation.

\section{References}

1. Savage DC. Microbial ecology of the gastrointestinal tract. Annu Rev Microbiol 1977; 31: 107-33.

2. Berg RD. The indigenous gastrointestinal microflora. Trends Microbiol 1996; 4: 430-5.

3. Backhed F, Ley RE, Sonnenburg JL, Peterson DA, Gordon JI. Host-bacterial mutualism in the human intestine. Science 2005; 307: 1915-20.
4. Eckburg PB, Bik EM, Bernstein CN, Purdom E, Dethlefsen L, Sargent M, et al. Diversity of the human intestinal microbial flora. Science 2005; 308: 1635-8.

5. Ley RE, Backhed F, Turnbaugh P, Lozupone CA, Knight RD, Gordon JI. Obesity alters gut microbial ecology. Proc Natl Acad Sci U S A 2005; 102: 11070-5.

6. Xu J, Gordon JI. Inaugural Article: Honor thy symbionts. Proc Natl Acad Sci U S A 2003; 100: 10452-9.

7. Bry L, Falk PG, Midtvedt T, Gordon JI. A model of host-microbial interactions in an open mammalian ecosystem. Science 1996; 273: 1380-3.

8. Hooper LV, Xu J, Falk PG, Midtvedt T, Gordon JI. A molecular sensor that allows a gut commensal to control its nutrient foundation in a competitive ecosystem. Proc Natl Acad Sci U S A 1999; 96: 9833-8.

9. Hooper LV, Wong MH, Thelin A, Hansson L, Falk PG, Gordon JI. Molecular analysis of commensal hostmicrobial relationships in the intestine. Science 2001; 291: $881-4$.

10. Backhed F, Ding H, Wang T, Hooper LV, Koh GY, Nagy A, et al. The gut microbiota as an environmental factor that regulates fat storage. Proc Natl Acad Sci U S A 2004; 101: 15718-23.

11. Xu J, Bjursell MK, Himrod J, Deng S, Carmichael LK, Chiang HC, et al. A genomic view of the humanBacteroides thetaiotaomicron symbiosis. Science 2003; 299: 2074-6.

12. Sonnenburg JL, Xu J, Leip DD, Chen CH, Westover BP, Weatherford $\mathrm{J}$, et al. Glycan foraging in vivo by an intestine-adapted bacterial symbiont. Science 2005; 307 : 1955-9.

13. Yoshida K, Shimizugawa T, Ono M, Furukawa $\mathrm{H}$. Angiopoietin-like protein 4 is a potent hyperlipidemiainducing factor in mice and inhibitor of lipoprotein lipase. J Lipid Res 2002; 43: 1770-2.

\footnotetext{
Jeffrey I. Gordon

Center for Genome Sciences

School of Medicine

Washington University

St Louis MO 63108

USA

E-mail: jgordon@wustl.edu
} 